\title{
4 Multimediale Elemente als Vermittlungsbausteine im Museum
}

Multimediale Elemente haben in Ausstellungen vor allem eine Aufgabe: Sie sollen dazu beitragen, Inhalte besser bzw. vertiefend zu erschließen. Die Umsetzung muss dabei vor allem mit Blick auf die verschiedenen Besuchergruppen und digitalen Nutzertypen erfolgen (vgl. Kap. 2.2.2). Medienkonzepte berücksichtigen heute nicht nur unterschiedliche Nutzerkompetenzen, sondern beinhalten auch ein intuitives Angebot, das unterschiedliche Rezeptionsgewohnheiten bedient.

In der Kommunikations- und Medientheorie herrschte lange Zeit die Auffassung vor, dass Informationen vor allem »relevant« und "glaubwürdig« sein müssen, um vom Rezipienten akzeptiert zu werden. Beide Eigenschaften sind für die Arbeit im Museum selbstverständlich. Kommunikationspsychologisch gesehen müssen Informationen heute aber auch »emotional nachvollziehbar« sowie »personalisierbar« sein. Eine weitere Grundlage gängiger Konzepte basiert zudem auf der Vorstellung einer asynchronen Informationsvermittlung - also einer zeitlich versetzten Kommunikation, etwa ein Ausstellungsbesuch und seine anschließende Nachbereitung mit dem Ausstellungskatalog. Die dialogische Konzeption, die gerade über die sozialen Medien das Meinungsbild und die Kreativität des Publikums mit einbezieht, basiert jedoch auf synchronen, also zeitgleichen Informationsstrukturen. Für immer weitere Teile des Publikums ist das aktive Teilen (Sharing) von Fotografien und Kommentaren auf Plattformen wie Facebook, Twitter oder Instagram ein fester Bestandteil des Museumsbesuchs (vgl. Kap. 3). So werden Informationen mit den unmittelbaren Erfahrungen der Besucher verknüpft und mit der subjektiven Gegenwart verwoben. Bei der Planung entsprechender Instrumente kommt also dem Zusammenspiel von Objektivität der Darstellung, Besuchererwartung, individueller Wahrnehmung und sinnlicher Erfahrung eine besondere Rolle zu. Ging man früher in der medienpädagogischen Arbeit davon aus, dass sich die von den Medien transportierten Informationen zum Exponat konkurrierend oder bestenfalls ergänzend verhalten', so entsteht durch die verstärkende Wirkung von digitalen Medien sowie durch den oben beschriebenen "Sharing-Effekt» ein ganz anderes Nutzer- bzw. Rezeptionsverhalten. Zwei grundlegende Fragen, die bei der Einbin-

1 Vgl. hierzu u. a. Schäfer, Denis: PDA: Mobiles Informationssystem für die Besucherbetreuung im Museum (Mitteilungen und Berichte aus dem Institut für Museumsforschung 43), Berlin 2007, www.smb.museum/fileadmin/website/Institute/Institut_fuer_Museumsforschung/Publikationen/Mitteilungen/MIT043.pdf oder auch Bienert, Andreas: Wie werden die neuen Medien die Optionen der Museen verändern?, in: Schuck-Wersig, Petra/Wersig, Gernot (Hrsg.): Deutsche Museen im Internet (Mitteilungen und Berichte aus dem Institut für Museumsforschung 13), Berlin 1998, S. 66-68, www.smb.museum/fileadmin/website/ Institute/Institut_fuer_Museumsforschung/Publikationen/Mitteilungen/MIT013.pdf 
dung von digitalen Medien in Museen beantwortet werden müssen, sind deshalb: Was will ich vermitteln? Und: Welche Erfahrungsräume der Nutzer sollen mit einbezogen werden?

Die Bandbreite an verfügbaren Medien ermöglicht den Kuratoren, Vermittlern und Medienplanern, das Objekt in seiner Mehrdimensionalität bzw. Multiperspektivität den Besuchern nahezubringen. Dabei entstehen unterschiedliche Kontextualisierungen: Während eine 3D-Darstellung oder 360-Grad-Ansicht das Objekt in seiner Gesamtheit zeigt, kann ein begleitender Text den historischen Kontext oder einen bestimmten Aspekt des Exponats, wie etwa den Gebrauchszusammenhang oder die Provenienz bzw. Objektbiografie, fokussieren. Durch die Einbindung von Animationen oder Bewegtbildern lassen sich zusätzlich komplexe Sachverhalte wie Funktionsweise oder Herstellungsart eines Objekts verständlich darstellen.

Angesichts dieser Vielzahl an möglichen Informationen wird in Fachkreisen die These einer medialen Überforderung des Betrachters diskutiert. Gerade vor diesem Hintergrund sind reflektierte Konzepte wesentlich, die das jeweilige Vermittlungsziel stets fest im Auge behalten. In keinem Fall bedeutet ein digitaler Ansatz automatisch eine bessere Vermittlung. Und nur zu oft werden die Vorstellungen einer effektiveren Vermittlung an junge Zielgruppen automatisch mit dem Einsatz digitaler Instrumente assoziiert. Was richtig sein kann, aber nicht muss.

Versteht man Museen als Orte des informellen Lernens (vgl. Kap. 1.2.1), dann müssen Informationen so aufbereitet werden, dass sie von den verschiedenen Lerntypen ${ }^{2}$ rezipiert werden können und idealerweise in den Wissensschatz der Besucher eingehen. Allerdings stehen Museen hier im Spannungsverhältnis zwischen kultureller Freizeitgestaltung, zunehmender Erlebnisorientierung und individualisierten Zugängen zu Wissen und Informationen. Der Museumsbesuch dient oft nicht vorrangig dem eigenen Bildungs- oder Wissenserwerb, sondern ist Bestandteil einer (touristischen) Besichtigungstour oder eines mit Freunden oder der Familie gestalteten Ausflugs. Gerade die Informationsvermittlung an Familien stellt eine besondere Herausforderung dar. Zwar sind vor allem Kinder und Jugendliche fasziniert von neuen Medien und möchten sie gerne ausprobieren, aber die Anwendung muss hier den unterschiedlichen Informationsbedürfnissen, Nutzungsgewohnheiten und Aufmerksamkeitsspannen aller, also der Kinder und der Erwachsenen, gerecht werden. Mithilfe der medialen Aufbereitung von Informationen können die unterschiedlichen Motivationen und Rezeptionsgewohnheiten heterogener Besuchergruppen jedoch gut bedient werden. Informationsvielfalt ist, sofern zielgruppenorientiert konzipiert, weniger als Informationsflut, sondern vielmehr als Chance zu verstehen.

\footnotetext{
2 Vgl. »Lerntheorien: Wie lernen Erwachsene? « in: Deutscher Museumsbund e. V. (Hrsg.): Museen und Lebenslanges Lernen - Ein europäisches Handbuch, Berlin 2010, S. 21-30, https://www.ne-mo.org/fileadmin/Dateien/public/topics/Learning/LifelongLearning inMuseums_DE.pdf
} 
Welches Exponat einen Besucher anspricht, hängt unter anderem von dessen Interessen, seinem Vorwissen, aber ebenso von der visuellen Attraktivität des Objekts und seiner Inszenierung ab. Letztere kann durch die Mittel der Gestaltung (Licht, Szenografie etc.) sowie durch den gezielten Einsatz von Medien gesteigert werden. Hierzu gehören etwa eine begleitende Medienstation oder die Einbeziehung des Objekts in die Führungslinie eines $\rightarrow$ Medienguides. Digitale Medien sind immer auch als kuratorisches Instrument zu verstehen, das das Rezeptionsverhalten der Museumsbesucher maßgeblich mit beeinflusst.

Für Kuratoren, technische Entwickler, Mediengestalter, Grafiker, Medienpädagogen und Vermittler bedeutet die Konzeption von Medienstationen oder Medienguides eine vielfältige gemeinsame Herausforderung. Diese geht weit über die technischen Aufgabenstellungen hinaus. Zielgruppenorientierung, Darstellungsvarianten, Grafik, Textlänge in Hinblick auf die jeweiligen Displaygrößen sind nur einige der zu bedenkenden Aspekte.

Die Einbeziehung von digitalen Medien bedeutet in der Regel auch finanziell eine größere Investition für ein Museum. Diese beschränkt sich nicht nur auf das eigentliche Produkt, sondern erfordert zuweilen auch eine flankierende digitale Infrastruktur für den erfolgreichen Betrieb, zum Beispiel kostenloses $\rightarrow$ Wi-Fi/ WLAN $^{3}$, wenn eine Anwendung auch vor Ort heruntergeladen werden soll. Fest installierte Anwendungen benötigen Strom- und Datenleitungen oder aufwendigere Einbauten (vgl. Kap. 5). Vielen Museen stehen bei der Planung entsprechender digitaler Systeme nicht nur finanzielle, sondern auch verwaltungsrechtliche oder denkmalpflegerische Probleme im Weg. Kabellose Übertragungssysteme, wie beispielsweise $\rightarrow$ Access Points, bieten bei Letzteren zumindest in Teilbereichen Lösungsmöglichkeiten.

Investitionsbedarf besteht aber nicht nur bei der notwendigen technischen Infrastruktur oder der Beschaffung grundlegender Hardware. Ein guter Budgetplan berücksichtigt Folgekosten, die unter anderem für die Wartung der Geräte, Update der Software oder das Erstellen bzw. Einpflegen neuer Inhalte anfallen können. Eine Orientierung über bereits im Haus genutzte technische Medien und etablierte Wartungsprozesse kann hier hilfreich sein. Hinzu kommt, dass häufig schon die in der Planungsphase anfallenden Kosten unterschätzt werden. Die Erfahrung zeigt, dass bei vielen Projekten die notwendigen Entwicklungszeiten, von der Konzeption bis zum fertigen Produkt, viel zu kurz angesetzt werden. Der Prototyp einer Anwendung ist lediglich ein Meilenstein im Entwicklungsprozess und oft wird für die Endproduktion noch einmal die gleiche Zeitspanne benötigt wie für die Prototypentwicklung.

3 Die viel diskutierte Störerhaftung wurde im Oktober 2017 für Betreiber von WLANNetzen abgeschafft, www.bundesrat.de/DE/plenum/plenum-kompakt/17/960/960-node. html\#top-10 
Im Budgetplan sollten neben Anschaffungs- und Herstellungskosten auch die Folgekosten veranschlagt werden: Wartung der Geräte, Update der Software, Einpflegen neuer Inhalte.

Bei der Neueinführung eines digitalen Angebots sollte auch eine Schulung der Mitarbeiter eingeplant werden. Das Museumspublikum benötigt unter Umständen Hilfestellung bei der Nutzung einer Anwendung, und die Institutionen sollten generell Bereitschaft zeigen, eine geeignete Informations- und Supportstruktur anzubieten. Dabei sollte nicht nur das Personal im Foyer mit den jeweiligen Funktionen der angebotenen Medien vertraut sein, sondern etwa auch die Aufsicht in den einzelnen Ausstellungsräumen. Kein Besucher läuft gerne zum Eingangsbereich zurück, wenn er mit einer Anwendung nicht zurechtkommt, sondern wird sich immer zuerst an die Mitarbeiter vor Ort wenden.

Bei der digitalen Vermittlung im Museum wird unterschieden zwischen Medienstationen, die zur Erschließung eines Objekts bzw. Themas stationär in der Ausstellung beitragen, und Medienguides (Audio- und Multimediaguides, vgl. Kap. 4.1), die als mobiles Führungssystem in einem größeren Zusammenhang oder themen- bzw. zielgruppenspezifisch (Highlightführung, Familienführung, spezielle Themenführung) durch das Museum leiten sowie als Lernanwendung für Schulklassen fungieren. Für einen erfolgreichen Einsatz sollten Stationen wie Guides gleichermaßen in das Bildungs- und Vermittlungskonzept des Museums eingebunden sein. Werden digitale Medienangebote erst nachträglich in eine Ausstellungssituation eingefügt, müssen Präsentationskonzepte womöglich überarbeitet werden. Digitale Medien sollen einen Mehrwert darstellen und dürfen kein Selbstzweck sein. Daher gilt es stets zu überlegen, welches Thema oder Objekt sich wirklich für eine multimediale Aufbereitung eignet.

Digitale Medien sollten stets im Rahmen eines Gesamtkonzepts und keinesfalls als Insellösungen eingesetzt werden.

Ein unerlässliches Thema bei jeder Art von Medieneinsatz sind neben den Urheber-, Nutzungs- und Persönlichkeitsrechten, die bei der Verwendung von Bild-, Ton- und Videomaterial beachtet werden müssen, auch die Impressums- sowie die Datenschutzpflicht. ${ }^{4}$

$4 \mathrm{Zu}$ allgemeinen Informationspflichten bei geschäftsmäßigen Telemedien siehe § 5 Telemediengesetz (TMG), www.gesetze-im-internet.de/tmg/BJNR017910007.html 
https://link. bsb-muenchen.de/ mbxix013

\subsection{Audio- und Multimediaguides}

Ein $\rightarrow$ Medienguide kann einen Museumsbesuch sinnvoll unterstützen und einzelne Themen gezielt vertiefen sowie Mehrsprachigkeit und $\rightarrow$ barrierefreie Alternativen anbieten. Er dient der Orientierung innerhalb einer Ausstellungseinheit und erlaubt es den Museumsbesuchern, im eigenen Tempo der individuellen Führungslinie zu folgen. Idealerweise wird es den Nutzern überlassen, die gewünschte Vertiefungsebene zu wählen und zu entscheiden, wie lange und wie intensiv sie sich mit einem einzelnen Objekt bzw. einem Thema auseinandersetzen möchten. Hierfür ist es essenziell, bereits im Vorfeld der Produktion zu entscheiden, welche Informationen obligatorisch und welche optional als Vertiefungsebene angeboten werden sollen. Wichtig ist auch die Art der jeweiligen Darstellung. Nicht jede Information muss in einem Text vorgehalten werden. Manche Zusammenhänge lassen sich besser mit einer Grafik, einem Film oder einer 3D-Darstellung veranschaulichen.

International gehören Führungssysteme bereits seit den 1970er Jahren - damals noch als Audioguide in Form eines Kassettenspielers - in Sonderausstellungen und Museen zum Standard. ${ }^{5}$ Mittlerweile zeichnet sich ab, dass der klassische Audioguide zwar technisch abgelöst bzw. inhaltlich durch Multimedia erweitert wird, jedoch steht der breite Einsatz von Multimedia im Museumsbereich (in Deutschland) noch aus. Ursachen hierfür liegen im merklichen Kostenaufwand und in einer gewissen Orientierungslosigkeit bei der Wahl des passenden Systems sowie bei der Einschätzung der eigenen Anforderungen und Bedarfe.

Die Entscheidung für ein spezifisches Ausgabegerät beispielsweise bestimmt nicht nur die Art der Kommunikation mit dem Besucher, sondern auch die Wahl der geeigneten Medien sowie die Umsetzung der Inhalte. So muss in einem ersten Schritt - nach Klärung der möglichen technischen Infrastruktur - der gewünschte Funktionsumfang definiert werden, um im Anschluss ein passendes Ausgabegerät (PDA-System, Tablet, Smartphone oder $\rightarrow$ Wearable), das zugehörige $\rightarrow$ Betriebssystem (u. a. iOS, Android oder Windows) und das geeignete Sender-Empfänger-System (unter anderem $\rightarrow$ QR-Code, $\rightarrow$ Beacons, $\rightarrow$ RFID) auswählen zu können. Weiterhin ist festzulegen, ob Leihgeräte zur Verfügung gestellt werden und/oder die Museumsbesucher ihre eigenen Geräte vor Ort nutzen sollen ( $\rightarrow$ BYOD). Die Entscheidung für Leihgeräte bringt auch immer die Frage nach Ausgabe- und Wartungsworkflows, aber auch zum Thema Diebstahlschutz mit sich. BYOD hingegen bedarf mitunter besonderer inhaltlicher und datenschutzrelevanter bzw. urheberrechtlicher Konzepte, da die ausgelieferten Inhalte auf den privaten Geräten des Publikums dargestellt werden.

Die Nutzung von Audio- und vertonten Videobeiträgen erfordert den Einsatz von Kopf- bzw. Einohrhörern. Diese ermöglichen es den Besuchern, ungestört zuzuhören und verhindern gleichzeitig eine Intensivierung der Geräuschbelastung in den

5 Vgl. Kunz-Ott, Hannelore (Hrsg.): Mit den Ohren sehen. Audioguides und Hörstationen in

Museen und Ausstellungen (MuseumsBausteine 14), Berlin/München 2012. 
Ausstellungsräumen. Kopfhörer schirmen die Besucher komplett von ihrem Umfeld ab. Das erhöht die Konzentrationsfähigkeit, unterbindet aber gleichzeitig einen direkten Austausch mit Begleitpersonen und mindert somit das Gemeinschaftserlebnis eines Ausstellungsbesuchs. Einohrhörer wiederum bieten diese Möglichkeit, sind aber oft problematisch in ihrer Handhabung. Bei der Anschaffung sollte daher auf Modelle mit elastischen »Bügeln« geachtet werden, die es erlauben, den Hörer sowohl am rechten als auch am linken Ohr zu tragen. Setzt das Museum darauf, dass die Besucher ihre eigenen Smartphones benutzen, sollten Einweg-Hörstöpsel vorgehalten werden. Kopf- und Einohrhörer müssen nach jeder Benutzung aus hygienischen Gründen gereinigt bzw. ihre Schaumstoffüberzüge ausgewechselt werden.

Wichtig ist, bei den Hörern auf eine ausreichende Kabellänge zu achten. Auch die Umhängebänder der Medienguides sollten Tragekomfort gewährleisten, also nicht im Schritt baumeln, aber auch den Blick auf das Gerät problemlos ermöglichen. Hier ist eine einfache Justiermöglichkeit der Gurtlänge hilfreich. Und natürlich muss die Aufhängung so angebracht werden, dass die Nutzer den Bildschirm in der korrekten Ansicht - und nicht auf dem Kopf stehend - sehen.

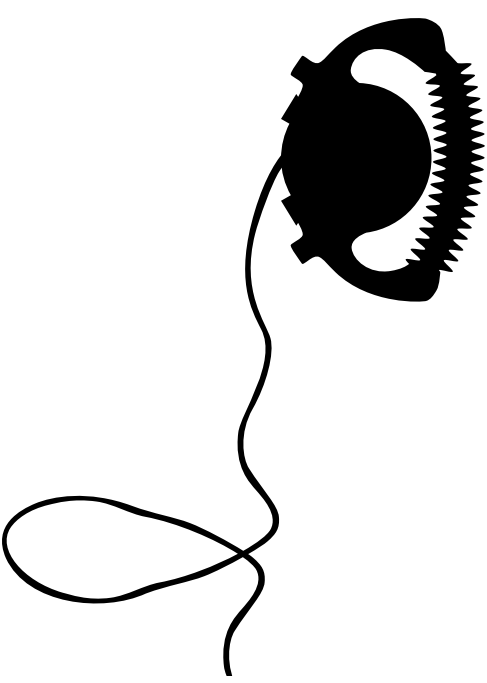

Abb. 1 Modell eines beidseitig tragbaren Einohrhörers, der mithilfe eines elastischen Bügels am Ohr befestigt wird. Grafik: Landesstelle für die nichtstaatlichen Museen in Bayern/ Julia Neller/CC BY 4.0 (https://creativecommons.org/licenses/by/4.0)

\section{Testen Sie im Selbstversuch Ihre Geräte auf Praktikabilität.}

\subsubsection{PDA-Systeme}

PDA-Systeme (Personal Digital Assistant), kleine tragbare Computer, nutzen ein in sich geschlossenes System, das, einmal professionell gestaltet und programmiert, lange nutzbar ist. Die Kosten für die Verwendung von PDA-Systemen sind gut kalkulierbar, denn eine Anpassung an immer neue Soft- und Hardwarevarianten ist in der Regel nicht notwendig. Andererseits ist meist der Abschluss eines Wartungsvertrages mit dem herstellenden Dienstleister notwendig, da diese Systeme normalerweise nicht von den Museumsmitarbeitern redaktionell bearbeitet werden können. Aktualisierungen sind nur bedingt bzw. umständlich möglich und kaum zeitnah umzusetzen. Diese Schwerfälligkeit dürfte beim Publikum wenig Begeisterung auslösen: Eine zunehmend digitalisierte Gesellschaft, die an komplexe Geräte wie die aktuellen Smartphones gewöhnt ist, erwartet auch in den Museen ein anspruchsvolles Niveau bei Technik und Gestaltung. 
Leihgeräte müssen aus hygienischen Gründen nach jeder Nutzung gereinigt werden. Und auch für ihre technische Instandhaltung und Wartung benötigen Sie personelle Ressourcen.

\subsubsection{Tablets und Smartphones}

Der Vorteil von Tablet- und Smartphone-Systemen (Smart Mobile Devices) als $\rightarrow$ Medienguides liegt in der einfachen Handhabung der Geräte, die den Besuchern zumeist schon vertraut und so schnell verständlich ist. Die Programmierung der Anwendungen (Apps) erfolgt für die gängigen $\rightarrow$ Betriebssysteme wie iOS, Android oder Windows, die, unter Einsatz von $\rightarrow$ Content-Management-Systemen, eine einfache Bearbeitung und Aktualisierung von Inhalten zulassen.

Auf der anderen Seite erfordern diese Betriebsumgebungen aber auch regelmäBige Updates, an die die App-Programmierung entsprechend angepasst werden muss. Anwendungen, die älter als zwei Jahre sind, können bereits hoffnungslos veraltet sein und sind auf Betriebssystemen neuerer Generationen womöglich nicht mehr funktionsfähig. Betriebsrelevante Systemupdates benötigen meist mehr Speicherplatz auf den genutzten Endgeräten und können dazu führen, dass andere speicherintensive Inhalte wie Videos oder Animationen nicht mehr funktionieren. Dies stellt Museen vor technische wie finanzielle Herausforderungen - ein Aspekt, der hinsichtlich der Planung der eigenen Apps berücksichtigt werden muss.

Technisch ist es heute einfach, einzelne Internetangebote in die Anwendungen einzubinden. Dies können Social-Media-Funktionen, der Zugriff auf die OnlineSammlung und vertiefende Information zu den Exponaten oder die Museumswebseite sein. Voraussetzung ist in der Regel die Verfügbarkeit eines geeigneten $\rightarrow \mathrm{Wi}-\mathrm{Fi}$ / WLAN im Haus. Die freie Verfügbarkeit eines Netzzugangs hat aber auch eine Kehrseite, denn die Nutzer können sich nach Lust und Laune im Netz bewegen. In jedem Fall bindet eine längere Recherche die Aufmerksamkeit des Betrachters und lenkt vom eigentlichen Exponat bzw. der Führungslinie ab.

\subsubsection{Wearable Technology}

Zu den derzeit möglichen digitalen Angeboten im Bereich der Vermittlung zählt auch die sogenannte Wearable Technology - tragbare Computersysteme, die in der Regel am Körper oder der Kleidung befestigt werden. Wearables werden hauptsächlich im Fitnessbereich und in der Gesundheitsvorsorge genutzt. Im Kontext eines Museums erscheinen sie, zumindest in Europa, noch ungewöhnlich, auch wenn es bereits erste Umsetzungsbeispiele gibt. ${ }^{6}$ Tatsächlich sind solche Anwendungen nur

6 Zum Einsatz von Wearables vgl. Bernstein, Shelley: A wearable future for the Barnes Foundation, Barnes Foundation - Medium, 19.10.2016, https://medium.com/barnesfoundation/a-wearable-future-for-the-barnes-foundation-8c4f57e4269e sowie Lex, Beate: Kulturvermittlung am Handgelenk, Blogeintrag MAK Blog, 7.2.2017, http://blog.mak.at/ smartwatch-app 


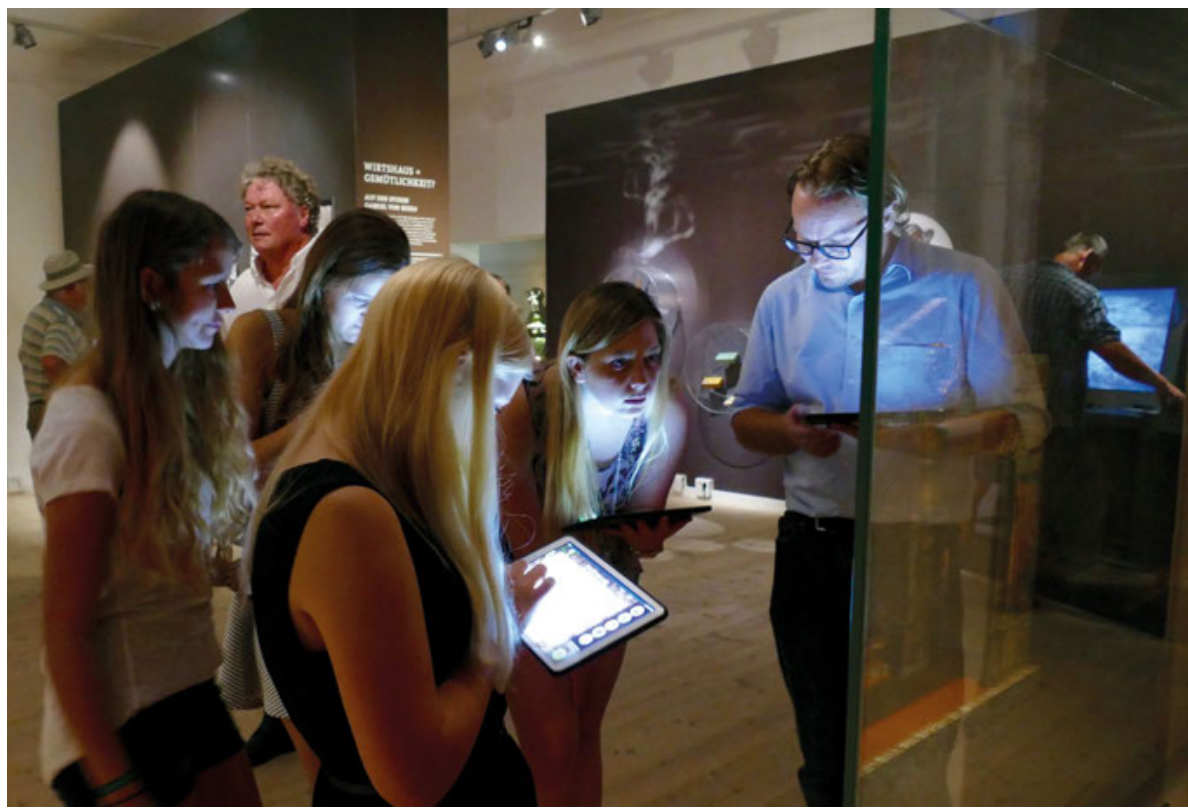

Abb. 2 In der Bayerischen Landesausstellung 2016 »Bier in Bayern« in Aldersbach wurde ein Medienguide mit dem Prototyp des App-Baukastens fabulAPP der Landesstelle für die nichtstaatlichen Museen in Bayern als Tablet-Rallye umgesetzt und auf Leihgeräten Schülern zur Verfügung gestellt. Foto: Haus der Bayerischen Geschichte, R. Ehm. Klier/innpressum

ansatzweise neu und seit der Erfindung von Walkman, Hörgerät oder dem Herzschrittmacher alltagstauglich.

Wearable Technology findet man unter vielen unterschiedlichen Bezeichnungen, beispielsweise als Fashionable Technology oder Tech Togs. Sie können dem $\rightarrow$ Internet der Dinge (Internet of Things, loT) zugeordnet werden, da sie als Alltagsgegenstände Teil einer vernetzten Infrastruktur sind. Die bekanntesten Wearables sind $\rightarrow$ Smartwatches sowie $\rightarrow$ Datenbrillen, die im Kontext von $\rightarrow$ Virtual und $\rightarrow$ Augmented Reality Verwendung finden (vgl. Kap. 7.1).

Der »NMC Horizon Report, 2013, Museum Edition« prognostizierte Wearables als eine für Museen relevante Technologie. ${ }^{7}$ Bis heute hat sich diese Prognose, zumindest im deutschen Sprachraum, jedoch nicht bestätigt. Der "personal.curator", eine Projektstudie des MAK - Österreichisches Museum für angewandte Kunst/Gegenwartskunst in Wien in Kooperation mit der Universität für angewandte Kunst Wien von 2017, die sich mit den partizipativen Einsatzmöglichkeiten von Smartwatches und $\rightarrow$ iBeacons in der Museumsvermittlung beschäftigte, zeigt exemplarisch die Vor- und Nachteile beim Einsatz dieser Technologie als $\rightarrow$ Medienguide auf. Insbe-

7 New Media Consortium Horizon Report, 2013, Museum Edition, www.nmc.org/ publication/nmc-horizon-report-2013-museum-edition 


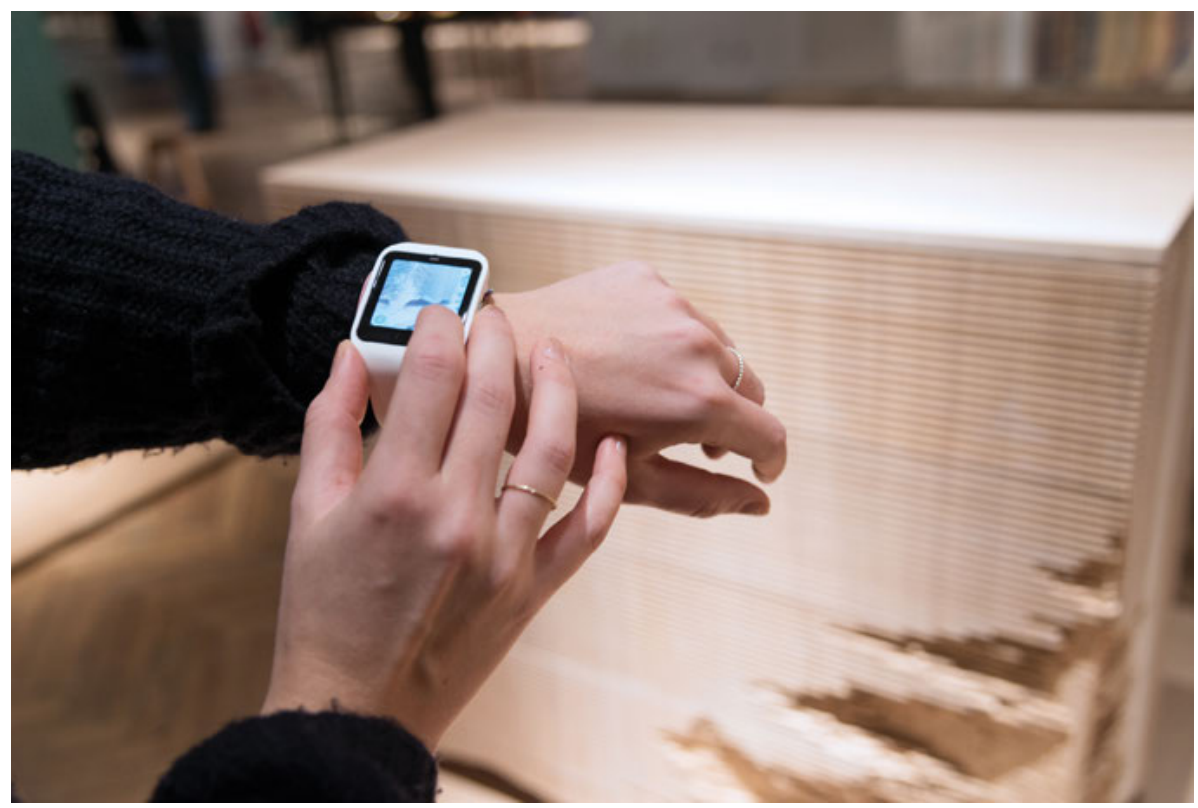

Abb. 3 Im Forschungsprojekt »personal.curator « des MAK - Österreichisches Museum für angewandte Kunst/Gegenwartskunst in Wien wurden die Einsatzmöglichkeiten von Smartwatches in der Kulturvermittlung untersucht. Foto: MAK/Mona Heiß

sondere die minimale Displaygröße stellte sich im musealen Vermittlungskontext als ein deutliches Manko heraus. Jedoch führte die Studie zu wertvollen Erkenntnissen über Interaktionsabläufe sowie die musealen und besucherorientierten Anforderungen an digitale Vermittlungssysteme, woran zukünftige Projekte sicherlich anknüpfen können. ${ }^{8}$

\subsubsection{BYOD - Bring Your Own Device}

Eine Alternative zum Leihgerät bietet das Konzept »Bring Your Own Device«, kurz BYOD. Eine Vielzahl der Besucher, die heute in ein Museum gehen, hat bereits ein eigenes Smartphone oder Tablet dabei. Zudem steigt die Bereitschaft, diese Geräte im Museum aktiv zu nutzen. Auf der Basis einer geeigneten Infrastruktur, dazu zählt unter anderem die Verfügbarkeit von $\rightarrow$ Wi-Fi/WLAN im Haus, und einer entsprechenden Anwendung eröffnen sich hier zahlreiche Möglichkeiten.

8 Die Studie ist bislang unveröffentlicht, kann aber im Einzelfall über die Universität für angewandte Kunst Wien angefragt werden. Im Ergebnis wurden Smartwatches zwar bei den Jugendlichen als technische Neuheit sehr gut angenommen, es gab aber auch gravierende Nachteile, neben der Displaygröße etwa auch die nicht immer intuitiv bedienbaren Geräte. Smartwatches werden im MAK wohl zukünftig nicht in der Vermittlung eingesetzt, www.mak.at/personalcurator 
Insbesondere bei BYOD sollten Museen darauf achten, dass digital basierte Vermittlungskonzepte nicht die einzige Option darstellen, um den Museumsbesuch medial zu begleiten. Idealerweise sind sie nur eine Facette im Gesamtportfolio und werden parallel durch weitere Angebote und die Ausgabe von Leihgeräten flankiert. Für ausländische Besucher stellt sich bei der Verwendung der eigenen Mobilgeräte immer noch die Frage nach möglichen Roaming-Kosten ${ }^{9}$, und wenig medienaffine Besucher kommen mit der technischen Umsetzung unter Umständen nicht zurecht oder verfügen über kein geeignetes Endgerät.

\subsection{Apps}

Im häufigsten Anwendungsfall werden $\rightarrow$ Medienguides als App angeboten. Apps (kurz für Applikationen) bezeichnen eine Anwendungssoftware für mobile Endgeräte und sind in fast allen Lebensbereichen zu finden. Am verbreitetsten sind Anwendungen für Smartphones und Tablets, welche für verschiedene Software-Plattformen entwickelt werden können. Die meisten Apps werden heute parallel sowohl für Android als auch für iOS geplant. Technisch unterscheidet man drei Varianten von Apps: native Apps, Web-Apps und hybride Apps.

Mobile Anwendungen im musealen Umfeld sind nicht unumstritten. Nach einem anfänglichen Boom der Technologie bleibt die Anzahl erfolgreicher Anwendungen bis heute überschaubar. Eine Vielzahl eher frustrierender Umsetzungen mit schlechten Downloadzahlen steht dem Wunsch vieler Museen nach einer eigenen App gegenüber. Mit Blick auf die internationale Bandbreite an möglichen Betriebs- und Vermittlungskonzepten lassen sich aber die Konturen erfolgreicher Anwendungen beschreiben. ${ }^{10}$

Wesentliches Qualitätskriterium einer App ist die Nutzerfreundlichkeit (Usability) (vgl. Kap. 6.1), welche ebenso wie eine einwandfreie technische Funktionsfähigkeit gewährleistet sein muss. Keinem Besucher sind lange Wartezeiten bis zum Download oder eine permanente Aktualisierung von Inhalten zuzumuten. Das Publikum wird rasch ungeduldig und möchte den Museumsbesuch schnell starten und ungestört vollenden. Auch sollten Apps nicht das gesamte Speichervolumen eines Smartphones belegen. Die Frustration und Verwirrung sind groß, wenn auch nach mehrmaligen Versuchen die App nicht funktioniert oder gar nicht erst geladen https://link. bsb-muenchen.de/ mbxix014

\footnotetext{
9 Seit dem 15.6.2017 dürfen laut EU MEMO/16/4396 Mobilfunkprovider keine zusätzlichen Kosten für Telefonate, SMS und verbrauchte Daten im EU-Ausland mehr verlangen. Im außereuropäischen Ausland bleiben Roaminggebühren weiterhin bestehen, www.bundesnetzagentur.de/DE/Sachgebiete/Telekommunikation/Verbraucher/Weitere Themen/InternRoaming/EURoaming/EURoaming-node.html

10 Mit »fabulAPP - Baukasten für digitales Storytelling im Museum« hat die Landesstelle für die nichtstaatlichen Museen in Bayern ein Schulungs- und Softwarekonzept entwickelt, das Museen in die Lage versetzt, mobile Applikationen selbstständig und kostenfrei zu entwickeln und zu betreiben, www.fabulapp.de
} 


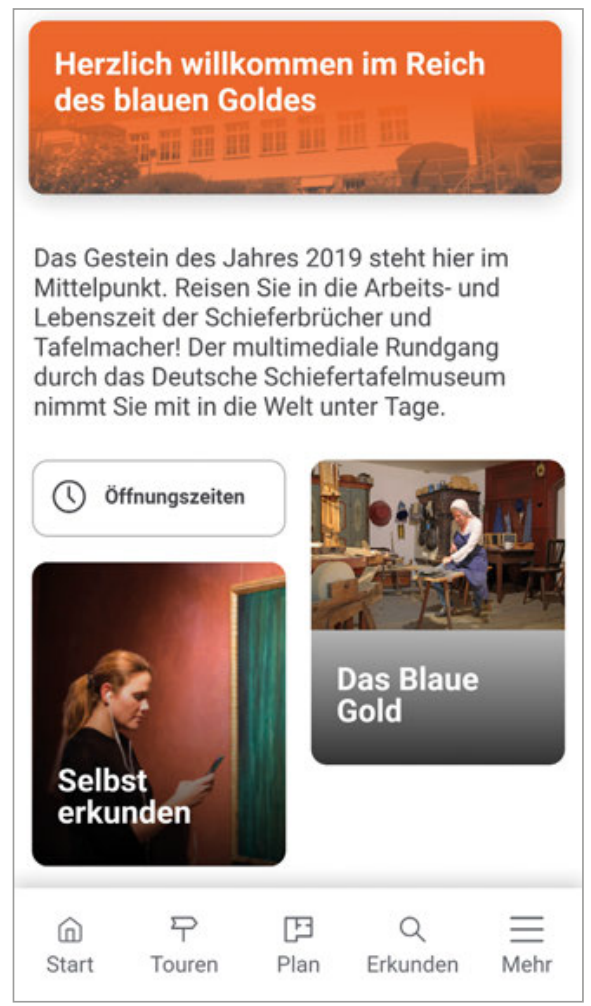

Abb. 4 Die App des Deutschen Schiefertafelmuseums Ludwigsstadt zeigt eine exemplarische Umsetzung mit fabulAPP (Screenshots).

werden kann. Auch eine richtige Vermarktung ist für den Erfolg einer App wesentlich. Um eine möglichst breite Wahrnehmung des digitalen Produkts zu erreichen, sollte die Anwendung nicht nur über die üblichen App Stores verfügbar und in das Kommunikationskonzept des Museums eingebunden sein, sondern auch vor Ort mittels Aufsteller und Flyer beworben werden. Auf jeden Fall sollte das Angebot auf der Webseite des Museums kommuniziert und per Link verfügbar gemacht werden. Denn im besten Fall hat der Besucher die App bereits im Vorfeld seines Besuchs auf sein eigenes Gerät geladen. Sollen die Nutzer die App vor Ort herunterladen können, so muss an ausreichend Raum und Rückzugsmöglichkeit gedacht sowie WLAN bzw. ein $\rightarrow$ Hotspot im Museum bereitgestellt werden.

\subsubsection{Native Apps}

Native Apps werden in der Regel über App Stores (u.a. Apple App Store, Google Play, Windows Phone Store) angeboten und vom Anwender direkt auf dem Gerät installiert, wo sie als Icon erscheinen. Sie funktionieren meist ohne direkte Netzanbindung und können offline im Museum, entsprechend der jeweiligen Konzeption 
und Programmierung, multimediale Inhalte verfügbar machen. Native Apps verzeichnen in der Umsetzung den höchsten Programmieraufwand, da sie für die jeweiligen $\rightarrow$ Betriebssysteme optimiert erstellt werden, im Gegenzug kann dann aber auch der Zugriff auf Hardware bzw. Funktionen des Endgeräts (wie Kamera, $\rightarrow$ GPS) in den Funktionsumfang integriert werden.

\subsubsection{Web-Apps}

Web-Apps sind wie (einfache) Webseiten konzipiert und benötigen weder einen App Store zur Bereitstellung noch aufwendige Softwarekomponenten, die von den Usern heruntergeladen und installiert werden müssten. Durch ihre betriebssystemunabhängige Konzeption sind sie meist weit kostengünstiger als native oder hybride Apps. Die User brauchen nicht mehr zu tun, als sich mit einem entsprechenden Netzwerk zu verbinden oder eine bestimmte Webadresse anzusteuern. Auf dieser werden dann alle Inhalte live eingespielt. Web-Apps sind für den Museumsbesuch besonders im Rahmen von $\rightarrow$ BYOD-Konzeptionen interessant und benötigen einen funktionierenden Internetzugang. Ohne eine ausreichende Netzanbindung in den jeweiligen Ausstellungsräumen kann kein Inhalt ausgeliefert werden. Als Alternative zu einer $\rightarrow$ Wi-Fi/WLAN-Bereitstellung durch das Museum bieten sich auch gekapselte WLAN-Netzwerke an, die auf der Basis von Local-Storage-Konzepten (lokale Speicherkonzepte) über entsprechende Signalgeber in einzelnen Räumen eingesetzt werden können. Die Daten werden quasi lokal zwischengespeichert und sind auch dann erreichbar, wenn keine Internetverbindung besteht.

Web-basierte Apps sind häufig an ein $\rightarrow$ Content-Management-System gebunden. Inhaltliche Updates sind daher unkompliziert und benötigen keine Kommunikations- oder Dienstleistungskette über eine Agentur oder einen Programmierer. Zusätzlich sind die webbasierten Inhalte im Netz auffindbar, wenn auch nicht über die App Stores zu vermarkten.

\subsubsection{Hybride Apps}

Hybride Apps verbinden native Apps mit Web-Apps. Sie werden in der Regel, für die Benutzer nicht sichtbar, innerhalb des Webbrowsers auf dem Endgerät betrieben. Auch sie müssen nicht für jedes $\rightarrow$ Betriebssystem (iOS, Android) einzeln entwickelt werden, sind also endgeräte- und plattformunabhängig. Zudem können auch hier inhaltliche bzw. technische Änderungen zügig eingespielt werden. Hybride Apps werden über die üblichen App Stores vertrieben bzw. verfügbar gemacht und sind daher mit einem Icon auf dem Endgerät vertreten. Im Gegensatz zu einer Web-App benötigen hybride Apps (je nach Programmieraufwand) Arbeitsspeicher auf dem Endgerät.

Welche dieser Varianten sich nun am besten zur Umsetzung eines geplanten App-Projekts eignet, kann anhand zentraler Entscheidungskriterien (Abb. 5) eingegrenzt werden. Grundsätzlich gilt: Soll eine sehr breite Zielgruppe angesprochen werden, spielt die Plattformunabhängigkeit eine wichtige Rolle; müssen Inhalte 
häufig aktualisiert werden oder steht nur ein kleineres Budget zur Verfügung, kommen eher eine Web-App oder (je nach gewünschtem Funktionsumfang) eine hybride App infrage; wird eine Anreicherung mit zahlreichen Funktionen und hohen Designansprüchen intendiert, ist vermutlich eine native App zielführend.

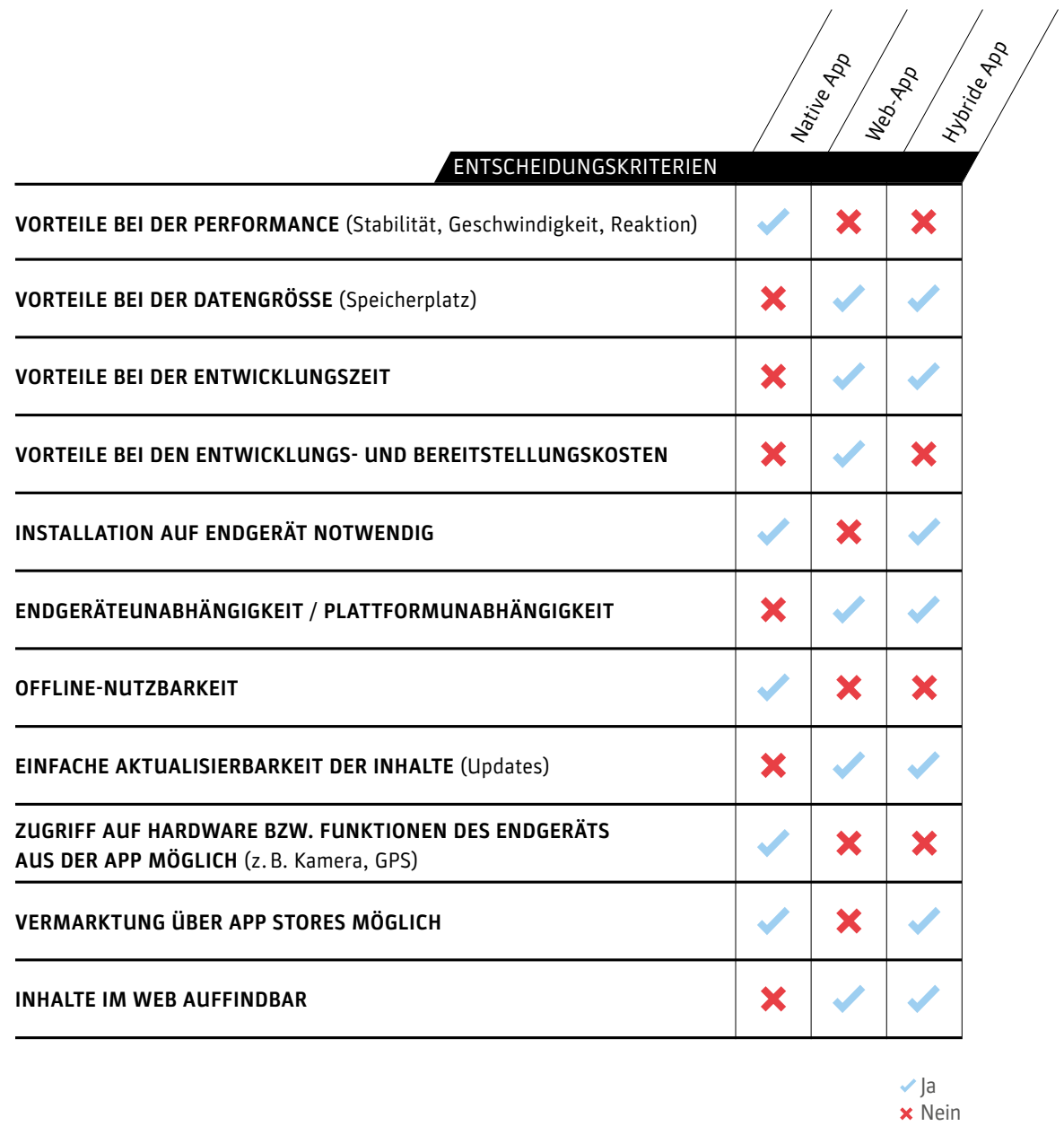

Abb. 5 Entscheidungskriterien bei der Planung einer App. Der Programmieraufwand bei einer hybriden App kann je nach Schwerpunkt recht unterschiedlich ausfallen. Grafik: Landesstelle für die nichtstaatlichen Museen in Bayern/Julia Neller/CC BY 4.0 (https://creativecommons.org/licenses/by/4.0) 


\subsection{Location-based Services (LBS) - standortbezogene Dienste}

https://link. bsb-muenchen.de/ Location-based Services (LBS) sind Dienste, die ausgewählte Informationen abhängig vom jeweiligen Standort auf einem mobilen Endgerät verfügbar machen. Dabei unterscheidet man zwischen proaktiven Anwendungen, die den Nutzern die Information automatisch zukommen lassen, und reaktiven Systemen, bei denen die Information angefordert werden muss, beispielsweise über die Aktivierung eines Funktionsbuttons, die manuelle Eingabe einer Objektnummer, das Scannen eines QR-Codes oder die Objekterkennung über die geräteeigene Kamera. Proaktive Systeme basieren in der Regel auf einem Positionsbestimmungsverfahren, wie etwa $\rightarrow$ GPS (Global Positioning System), Funk, Infrarot oder eine $\rightarrow$ WLAN-Infrastruktur, das den Standort des Endgeräts bzw. des Besuchers ermittelt: Egal, ob die Besucher einen Raum betreten, ein Objekt passieren oder vor einem Exponat zu stehen kommen, werden innen Informationen automatisch zugespielt.

Location-based Services werden bereits in zahlreichen Spiele-Apps, aber auch im touristischen Bereich eingesetzt, um den Nutzern etwa Informationen über standortnahe Sehenswürdigkeiten (Points of Interest), Restaurants, Einkaufsmöglichkeiten oder den öffentlichen Nahverkehr zu vermitteln. Im Museum unterstützen LBS-Anwendungen die Besucher bei der Orientierung im Gebäude bzw. Gelände und leiten sie auf Führungslinien oder an einen bestimmten Punkt.

Neben der Nutzung von GPS, das vorrangig im Außenbereich Einsatz findet, können Informationen innerhalb von Gebäuden über Systeme wie $\rightarrow$ RFID, $\rightarrow$ NFC oder $\rightarrow$ Beacons standortbezogen transportiert werden. Auf der Basis von Sender-EmpfängerSystemen werden RFID-Chips oder Beacons in Museen darüber hinaus auch zur Datensammlung bei Temperatur- bzw. Luftfeuchtemessung verwendet. QR-Codes hingegen kommen, neben dem altbekannten Barcode, häufig auch in der Inventarisierung und Dokumentation (Sammlungsmanagement) zum Einsatz.

\subsubsection{QR-Codes}

Der QR-Code (Quick Response) ist ein zweidimensionaler Code, der ursprünglich für die Autoindustrie entwickelt wurde. Das System ist robust (auch Fragmente der Bildmarke können ausreichen, um den Code technisch lesbar zu halten) und weit verbreitet. Der Code besteht aus einer quadratischen Matrix, die kodierte Daten binär darstellt. Die Besucher scannen beispielsweise mit der Kamera eines internetfähigen Handys einen Code und werden zum hinterlegten Inhalt weitergeleitet. Das kann ein Link auf eine Webseite, aber auch ein Audiofile oder ein Video sein.

Die Codes lassen sich über einfache QR-Code-Generatoren erzeugen." Zum Scannen wird bei älteren mobilen Endgeräten eine zusätzliche Reader-Software

11 Z. B. http://goqr.me/de oder www.qrcode-generator.de Darüber hinaus können bei sogenannten dynamischen QR-Codes die hinterlegten Inhalte beliebig editiert werden, während der einmal generierte QR-Code selbst unverändert bleibt. 

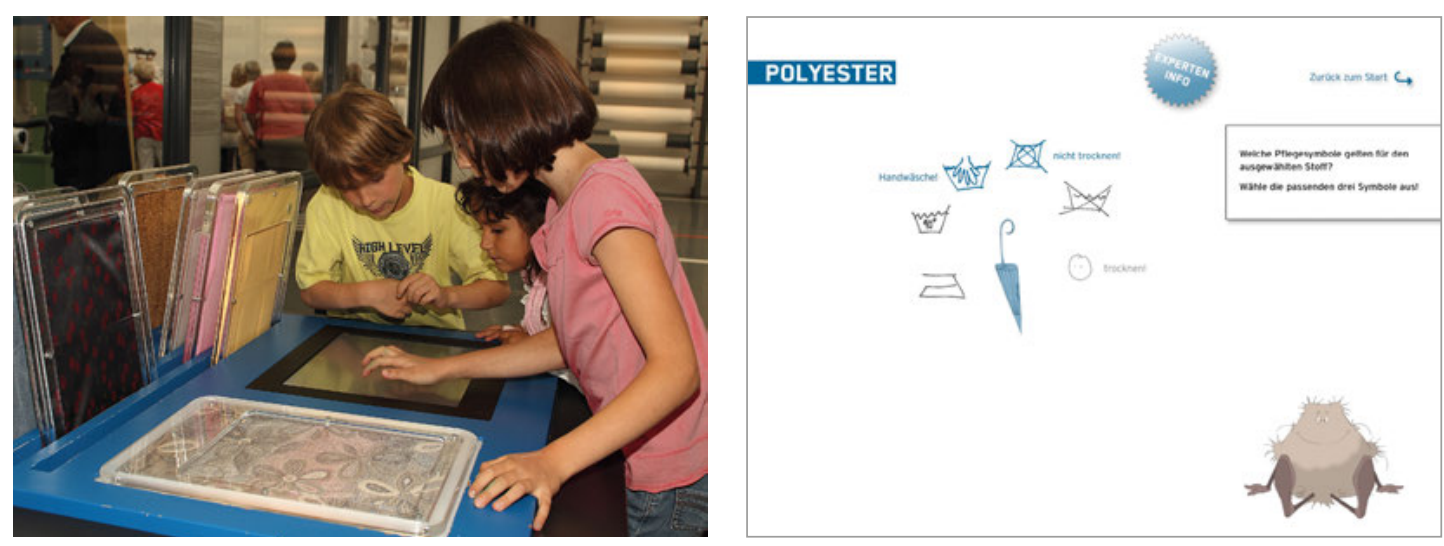

Abb. 6 Interaktiver Comic: Im tim - Staatliches Textil- und Industriemuseum Augsburg leitet eine Identifikationsfigur durch die Textilgeschichte. Stoffproben können auf einem Leuchttisch auf ihre Gewebeeigenschaften hin untersucht werden, gleichzeitig wird über eine eingebaute RFID-Technologie die Comicfigur »Fussel« am Touchscreen gesteuert. Fotos: Sascha Schneider/tim (links), 2av GmbH/tim (rechts)

(App) benötigt. Bei Smartphones und Tablets der neueren Generation ist diese Funktion bereits im Basissystem der Kamera verbaut. Darüber hinaus verfügen manche mobilen Browser über eine Scanfunktion - so beispielsweise Google Chrome.

\subsubsection{RFID - Radio-Identifikations-Systeme}

Unter RFID (Radio Frequency Identification) versteht man Radio-Identifikations-Systeme, die berührungslos Daten übermitteln. Zu dieser Technologie gehören immer ein RFID-Transponder und ein entsprechendes Lesegerät (Reader). Der Transponder besteht aus einem Mikrochip und einer Antenne, die mit dem Lesegerät kommuniziert. RFID-Transponder gibt es in vielen Varianten. Am bekanntesten sind Schlüssel, Chipkarten oder Labels. Die Reichweite dieser Mikrochips ist sehr unterschiedlich und beträgt üblicherweise 1,5 bis hin zu 10 Meter. Aktive Chips haben eine eigene Energiequelle, meist eine Batterie, über die sie betrieben werden. Passive Transponder dagegen werden über Funkwellen, etwa während des Lesevorgangs, mit Energie versorgt und haben meist eine geringere Reichweite.

RFID-Systeme werden beispielsweise in Bibliotheken bei der Ausleihe von Büchern oder in Museen zur Inventarisierung von Sammlungsbeständen eingesetzt. In Ausstellungen finden RFID-Systeme aber auch in Kombination mit $\rightarrow$ Medienguides Verwendung. So können beispielweise im Boden vor einem Exponat, an der Ausstellungswand oder dem Vitrinensockel RFID-Tags angebracht werden, deren Informationen über den Reader im Medienguide lesbar gemacht werden. RFID-Transponder bieten zahlreiche Möglichkeiten, Objekte mit Informationen, Bildern, Fotografien oder Filmen zu verknüpfen. In Verbindung mit einer Webseite oder einem 


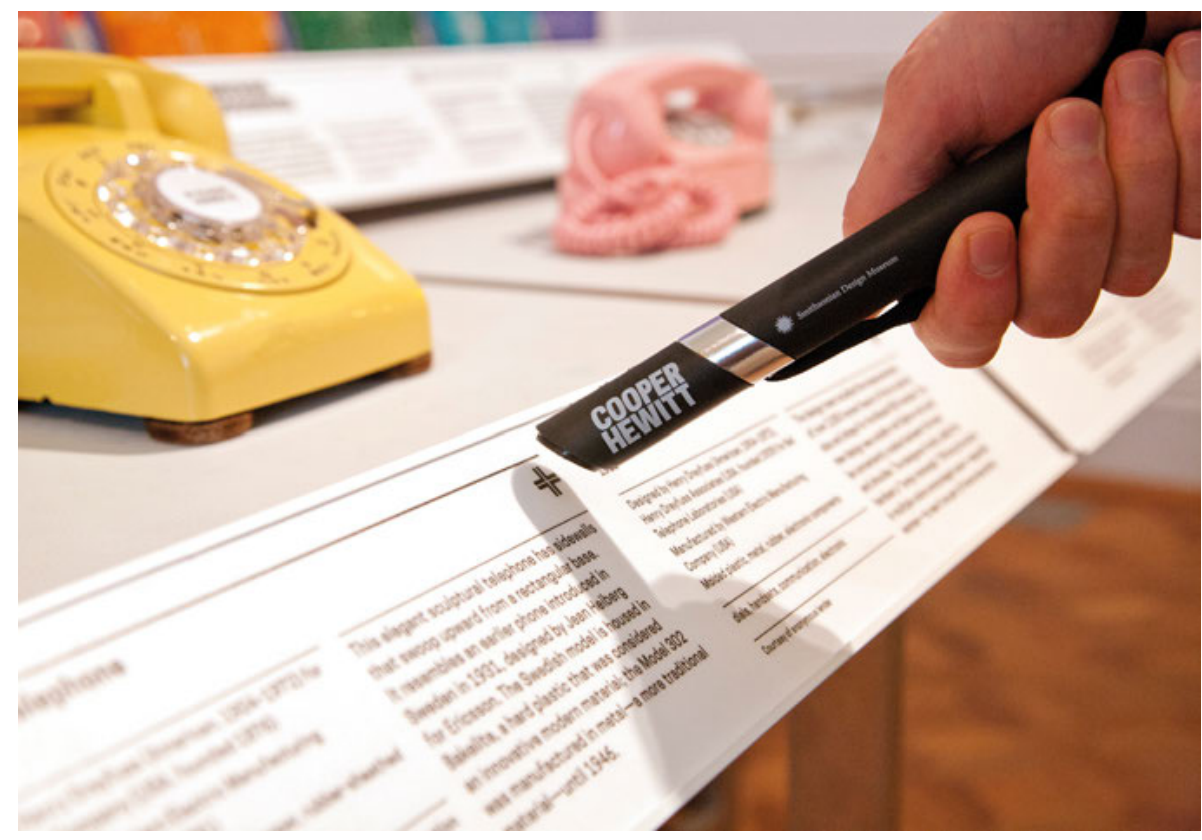

Abb. 7 »The Pen« des Cooper Hewitt Museums in New York basiert technisch auf der NFC-Technologie und ermöglicht den Besuchern eine direkte Interaktion mit der Sammlung über die Zeichnen- und Datensammel-Funktion auf den Medientischen. Foto: Matt Flynn (c) Smithsonian Institution

Mailingprogramm können Informationen, die im Museum gesammelt wurden, sogar mit nach Hause genommen werden.

\subsubsection{NFC - Near Field Communication}

Near Field Communication, also Nahfeldkommunikation, basiert im Wesentlichen auf der RFID-Technologie. Hierbei handelt es sich um einen kontaktlosen Austausch von Daten über eine kurze Distanz. Genutzt wird in der Regel das eigene (NFCfähige) Smartphone oder Tablet, aber auch die Integration der kleinen NFC-Etiketten in andere Endgeräte ist möglich. NFC wird häufig im Bereich des bargeldlosen Zahlungsverkehrs genutzt oder im Rahmen von papierlosen Eintrittskarten.

Im Museums- und Ausstellungsbereich bietet die NFC-Technologie den Nutzern die gleichen Möglichkeiten wie RFID-Anwendungen, nur dass hier keine Geräte ausgeliehen werden müssen, sondern das eigene Smartphone als Lesegerät benutzt werden kann. Da die Besucher keine App im Vorfeld herunterladen müssen, können sie während des Besuchs spontan entscheiden, ob sie auf zusätzliche Informationen zugreifen möchten. Auch hier können Daten auf dem Endgerät gespeichert und mit nach Hause genommen werden. Allerdings muss beim Einsatz von NFC darauf geachtet werden, dass nicht alle Geräte die gleiche Auflösung und Darstellungsfähigkeit haben und auch nicht jeder Besucher ein NFC-fähiges Gerät besitzt. 


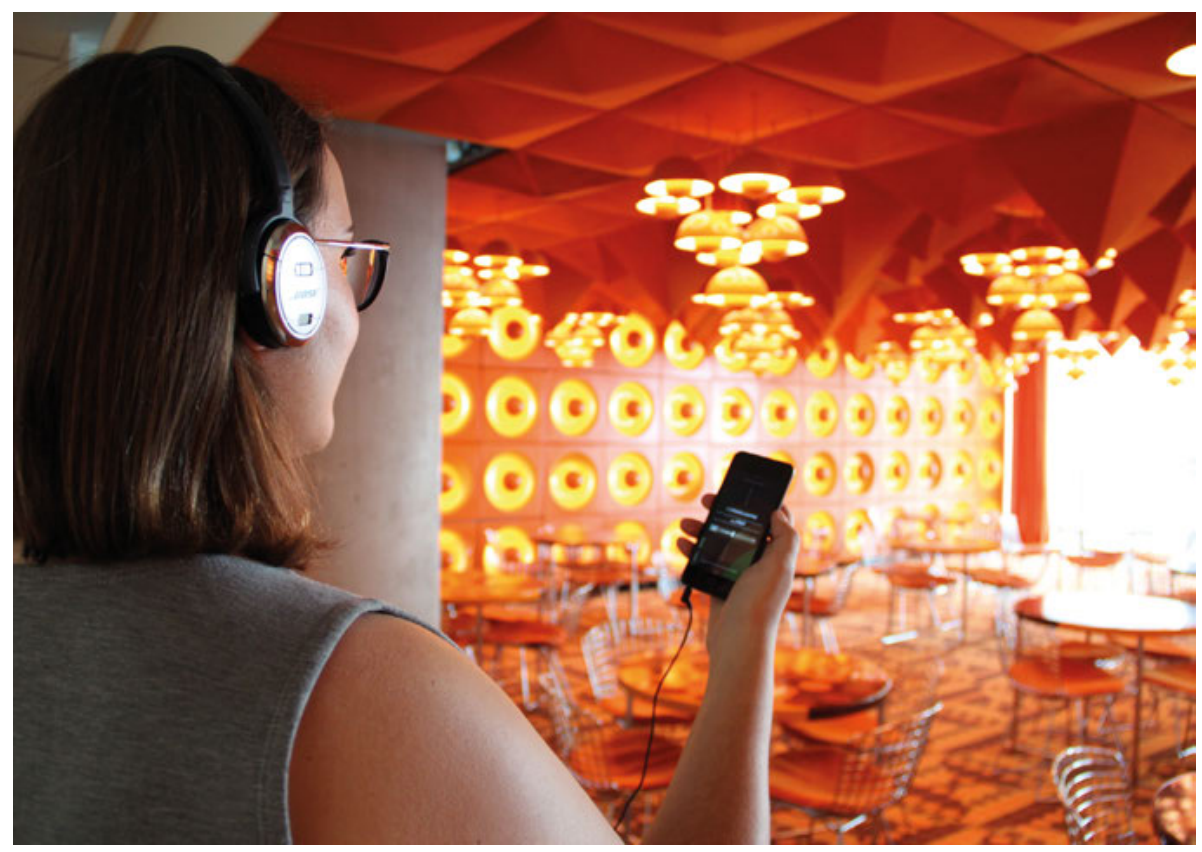

Abb. 8 Die interaktive Audiotour »Im Sog der Zeit« des Museum für Kunst und Gewerbe Hamburg verbindet zehn historische Epochenräume (hier: Verner Pantons SpiegelKantine) über eine App. Mithilfe von iBeacon-Sendern erkennt diese den Standort der Besucher und wählt den entsprechenden Audiobeitrag an. Foto: MKG

\subsubsection{Beacons}

Die Beacon-Technologie funktioniert wie auch RFID und NFC nach dem Sender-Empfänger-Prinzip. Sie ermöglicht Indoor- und Outdoor-Navigation sowie die Ausspielung ortsbezogener Informationen. Die batteriebetriebenen oder an das Stromnetz koppelbaren Beacon-Sender haben etwa die Größe einer Streichholzschachtel und können in Räumen oder direkt bei Objekten angebracht werden. Kommt ein Empfänger, und das kann ein einfaches $\rightarrow$ bluetoothfähiges Smartphone sein, in die Reichweite des Senders, wird der jeweilige Standort des Besuchers lokalisiert und der entsprechende Inhalt (über ein »Muttersystem«, etwa eine App) ausgeliefert.

Der häufig genutzte Markenname iBeacon wurde als proprietärer Standard 2013 von Apple eingeführt. Das Verfahren wird sowohl von iOS als auch von Android-Versionen unterstützt und basiert auf $\rightarrow$ Bluetooth Low Energy.

In der Praxis zeigt sich bei der Beacon-Technologie noch technischer Verbesserungsbedarf. Der zeitliche Aufwand zur richtigen Abstimmung der Signale in einem komplexeren räumlichen Gefüge, zumal wenn dieses von offenen Raumsituationen, Spiegelflächen (Vitrinen) und anderen »Störfaktoren« gekennzeichnet ist, bleibt für Museen eine deutliche Herausforderung. Derartige Schwierigkeiten bei der punktgenauen Lokalisierung der Empfänger, Überlagerungen bei den Signalen der Sen- 
der oder auch Signalstörungen in Abhängigkeit von der baulichen Struktur der Räume oder bei einer großen Anzahl von Besuchern bleiben noch zu lösende Parameter der eigentlich überzeugenden Technologie.

\subsection{Medienstationen}

Medienstationen können sich auf einzelne Objekte beziehen oder ganze Themenkomplexe behandeln. Letztere sind häufig im Eingangsbereich eines Museums oder einer Ausstellung positioniert und fokussieren meist weniger die Multiperspektivität einzelner Objekte, sondern beispielsweise einen regionalen, historischen oder thematischen Gesamtzusammenhang. Sind diese Informationen zu komplex gehalten oder ohne direkten räumlichen Bezug zu den Objekten, sehen sich die Besucher mit der Aufgabe konfrontiert, diese Fakten zu memorieren, in die Ausstellung "mitzunehmen« und dort den jeweiligen Exponaten zuzuordnen.

Medienstationen können Informationen in mehreren Vertiefungsebenen wiedergeben. Die Logik der Benutzerführung spielt dabei eine wesentliche Rolle: Die Besucher sollen dazu animiert werden, weitere Informationen abzurufen. Die Dauer der Informationsrecherche und die Reihenfolge des Abrufs entscheiden sie dabei selbst. Die Inhalte erreichen die Nutzer über verschiedene Medienformate - Texte, Bilder, Tonbeiträge, Videos, Grafiken oder 3D-Darstellungen -, die linear, also in festgelegter Reihenfolge, oder nicht linear angeordnet sein können. Im Gegensatz zum linearen Erzählen lebt das nicht lineare Erzählen davon, dass die Geschichte in einzelne Module zerlegt wird, die parallel in verschiedenen Medienformaten angeboten werden können.

Achten Sie darauf, Medienstationen nicht mit Inhalten zu überfrachten. https://link.

bsb-muenchen.de/ mbxix016

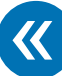

Medienstationen erreichen einen hohen Aufmerksamkeitswert und sollten entsprechend ökonomisch eingesetzt werden. Sie konkurrieren nicht nur mit den Objekten und Texttafeln, sondern wirken sich auch auf die Verweildauer im Museum bzw. in einzelnen Räumen aus, da sie die Aufmerksamkeit der Besucher binden. Das gilt insbesondere für interaktive Medienstationen. Diese sind auf einen Dialog zwischen Nutzer und Gerät ausgerichtet und animieren die Besucher zur eigenen Aktivität, etwa durch Spiele oder digitale Geschichten. Im Gegensatz zu Infoterminals ermöglicht diese Art von Medienstationen eine aktive Teilnahme und fördert so die Auseinandersetzung mit dem und das Verständnis für das Exponat.

Eine Untergruppe der interaktiven Medien sind die kollaborativen Medien. Hier geht es nicht nur um den Dialog zwischen Nutzer und Gerät, sondern auch zwischen den Nutzern selbst. Sie eröffnen so die Möglichkeit, Gemeinschaft zu erleben: Aus 


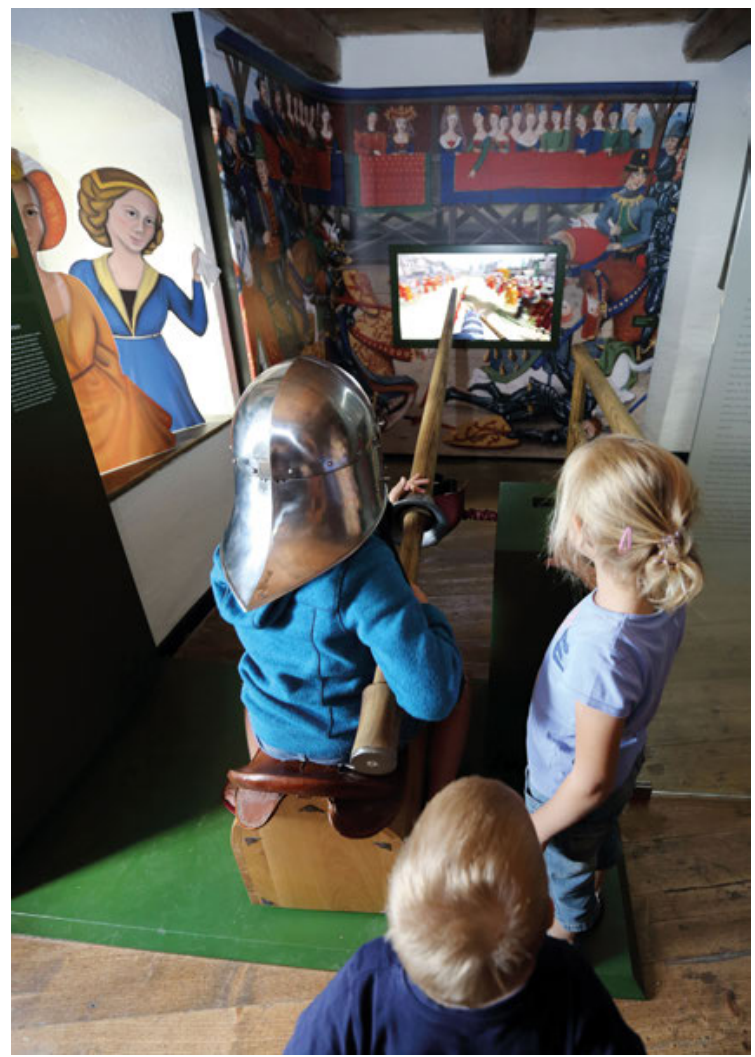

Abb. 9 Im Stadtmuseum

Burghausen können Besucher mithilfe eines Ritterhelms mit integrierter 3D-Brille gegen virtuelle Turniergegner antreten. Foto: Gerhard Nixdorf

Interaktion entsteht wirkliche Partizipation..$^{12}$ Im Bereich der $\rightarrow$ Serious Games kann über diese Medien gemeinsam gespielt oder eine Aufgabe gelöst werden. Wichtig ist dabei, dass die anderen Nutzer jeweils von den Ergebnissen der Vorgänger bzw. Mitnutzer profitieren können und ein Ziel gemeinschaftlich erreicht wird. In Museen sollen kollaborative Medien nicht nur Informationsangebote mit Interaktionen verknüpfen, sondern auch eine Gemeinschaftserfahrung vermitteln, um so die sozialen Interaktionen zwischen den Besuchern zu fördern. Oftmals ist das gemeinschaftliche Agieren an einer Medienstation jedoch auch mit einer Erhöhung des Geräuschpegels verbunden, was von anderen Besuchern als störend empfunden werden oder bei einer Führung zu Unterbrechungen führen kann. Dieser Aspekt sollte bei der Planung von Medienstationen und Führungslinien berücksichtigt werden.

12 Vgl. auch Schwan, Stephan/Trischler, Helmuth/Prenzel, Manfred (Hrsg.): Lernen im Museum: Die Rolle von Medien für die Resituierung von Exponaten (Mitteilungen und Berichte aus dem Institut für Museumsforschung 38), Berlin 2006, hier v. a. Kirchberg, Volker: Zur individuellen Konstruktion medialer Museumserlebnisse - Die Abhängigkeit der Vermittlung von eigenen Erfahrungen mit neuen Medien, S. 37-46, www.smb. museum/fileadmin/website/Institute/Institut_fuer_Museumsforschung/Publikationen/ Mitteilungen/MIT038.pdf 
Medienstationen sind besonders attraktiv und können mitunter zu Wartezeiten für Benutzer führen.

\subsubsection{Exkurs: Szenografie - Raumprojektion und Rauminstallation}

Szenografische Installationen gehören mit zu den interessantesten und anspruchsvollsten Varianten der Medienanwendung. Dabei werden die verschiedenen elektronischen Medien in ein künstlerisches Konzept eingebunden, in dem Licht, Ton, Film und Bild als Gestaltungselemente genutzt und aufeinander abgestimmt sind. Diese durchaus auch raumgreifenden Installationen sollten immer themenspezifisch geplant werden. Aufgrund des benötigten Aufwands eignen sie sich insbesondere für größere Ausstellungen oder sollten im Rahmen einer Neukonzeption der Dauerausstellung eines Museums in Betracht gezogen werden. Die Integration szenografischer Installationen in eine bestehende Ausstellung bedarf einer Erweiterung des Kommunikations- und Ausstellungskonzeptes, da sie erheblichen Einfluss auf die gesamte Wahrnehmung eines Themas haben. Neben der Auswahl der zu begleitenden Objekte gehört vor allem die Erarbeitung und Umsetzung eines auf den jeweiligen Raum abgestimmten Medienkonzepts zu den Herausforderungen in der Planung. Damit sich die verschiedenen Medien im Museum nicht untereinander Konkurrenz machen, muss dieses Medienkonzept auch die angrenzenden Räume mit einbeziehen.

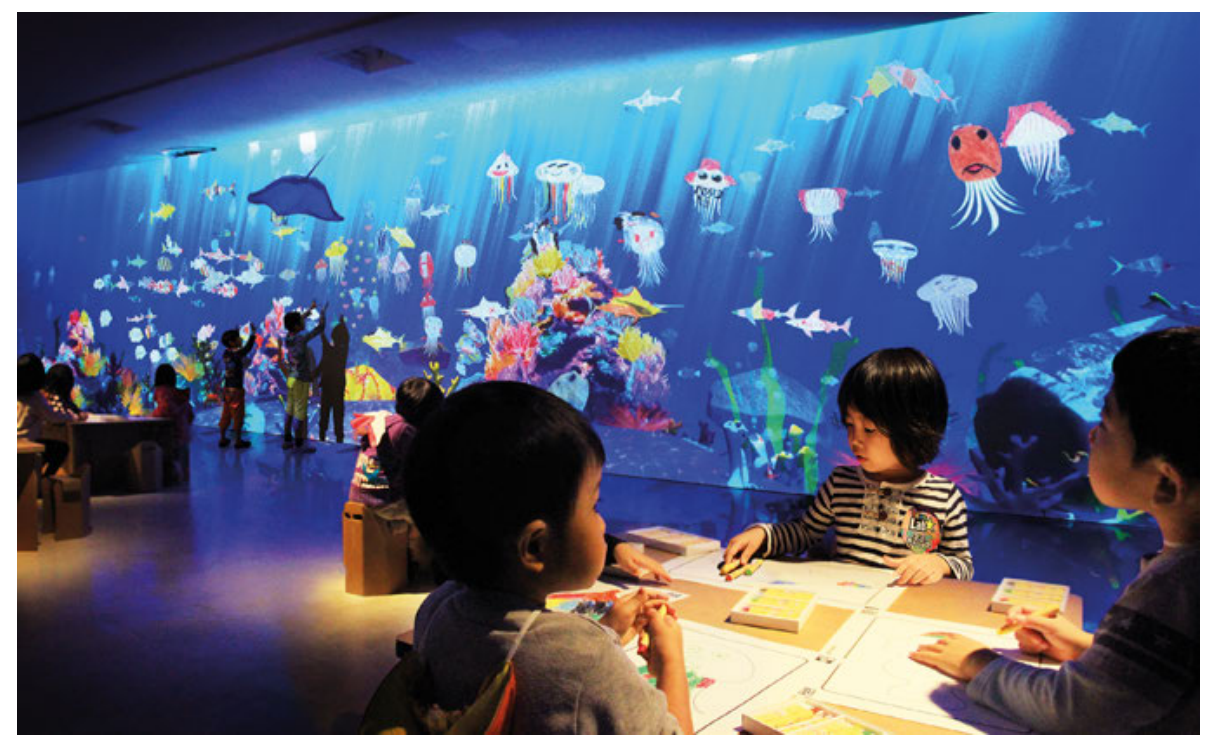

Abb. 10 In der »Future World« des ArtScience Museum, Singapur, erwachen eingescannte Zeichnungen zu interaktivem Leben. Foto: teamLab 
Rauminstallationen können Informationen unmittelbar erfahrbar machen und haben oft einen narrativen Hintergrund. Kuratoren, Ausstellungsgestalter und Medienplaner sollten sich daher im Vorfeld überlegen, welche Geschichte sie erzählen und welcher Kernaussage der meiste Erzählraum eingeräumt werden soll (vgl. Kap. 6.3). Szenografische Installationen emotionalisieren, sie sprechen die Gefühlsebene der Besucher an und sind notwendigerweise stark erlebnisorientiert. Ein Beispiel hierfür sind immersive Räume mit interaktiven Komponenten. Das ArtScience Museum in Singapur etwa entwickelte zusammen mit einem interdisziplinären Künstlerkollektiv regelrechte Multimedialandschaften. Hier können die Besucher beispielsweise eigene Zeichnungen einscannen, die dann umgehend als interaktives Objekt in die Raumprojektion aufgenommen werden.

\subsection{Fragen und Anregungen}

- Welchen Mehrwert stellen Ihre multimedialen Angebote für die Besucher dar? Medien dienen der Erweiterung des Vermittlungskonzepts. Sie sollten nicht zum Selbstzweck oder als Zeichen einer vermeintlichen Modernität genutzt werden, sondern immer einen Mehrwert darstellen.

- Wie sieht Ihre Zielgruppe aus?

Für die Erstellung und Umsetzung eines digitalen Medienkonzepts ist eine Analyse der möglichen Adressaten notwendig. Erstellen Sie eine Übersicht, in der Sie festhalten, welche Zielgruppe Sie mit welchem Medium ansprechen möchten.

- Welche Darstellungsform eignet sich wofür?

Durch die Verbindung von Text, Audio, Bild und Video lässt sich für jeden Inhalt das geeignete Medium finden. Funktionsweisen können durch Animationen veranschaulicht werden, Grafiken zeigen Zusammenhänge auf und 3D-Darstellungen ermöglichen einen Blick in das Innere des Exponats.

- Welche Vertiefungsebenen sind sinnvoll?

Ein Zuviel an Information kann die Besucher überfordern. Daher ist es wichtig zu überlegen, ob und wie viel Zusatzinformation das jeweilige Exponat bzw. Thema wirklich benötigt. Eine mit Informationen überfrachtete Anwendung ist selten hilfreich und die Frustrationsschwelle ist bei den Besuchern eher niedrig anzusetzen.

- Wie sehen die baulichen Voraussetzungen in Ihrem Haus aus?

Eventuell sind Um- und Einbauten nötig - deren Umsetzbarkeit sollte bereits im Vorfeld der Planung geklärt werden, auch in Hinblick auf eventuelle Vorgaben des Denkmalschutzes.

- Was muss bei der Budgetplanung beachtet werden?

Anwendungen werden oftmals nicht nachhaltig geplant. Gerne wird vergessen, dass neben der Entwicklung und Anschaffung von digitalen Produkten auch Folgekosten anfallen. Diese beinhalten Positionen für Betrieb (Software-Updates) 
und technische Wartung, aber auch Schulungen der Mitarbeiter oder eine redaktionelle Aktualisierung der Inhalte. Um Planungssicherheit zu gewährleisten, sollten diese Folgekosten vor allem bei Dauerausstellungen für einen Zeitraum von etwa fünf Jahren berechnet werden.

- Ist Ihr Personal ausreichend geschult?

Denken Sie daran, dass Ihre Besucher mit sehr unterschiedlichen technischen Kenntnissen in die Ausstellung kommen. Ihre Mitarbeiter sollten deshalb stets in der Lage sein, bei der Handhabung von Medien Hilfestellung zu leisten.

- Verfügt lhr Haus über die entsprechenden technischen Voraussetzungen? Definieren Sie die notwendige digitale Infrastruktur für den erfolgreichen Betrieb einer Anwendung. Dazu gehören ggf. nicht nur die Stromversorgung einer Medienstation, sondern auch flankierende Ressourcen wie WLAN oder das Bereitstellen eines $\rightarrow$ Hotspots zum Download eines digitalen Angebots.

- Wie sorgen Sie für die Rückbindung eines digitalen Angebots im eigenen Haus?

Anwendungen müssen nicht nur allen Fachabteilungen im Haus bekannt sein, sondern von diesen auch aktiv in die Vermittlungsarbeit eingebunden werden.

- Leihgeräte oder BYOD? Im besten Fall bieten Sie beides an, um keine Besuchergruppe auszuschließen.

- Was ist der geeignete Standort für eine Medienstation? Medienstationen ziehen erhöhte Aufmerksamkeit auf sich und sollten somit nie in direkte Konkurrenz mit den Exponaten treten. Gleichzeitig ist darauf zu achten, dass die Benutzer der Stationen die Führungslinien nicht blockieren. 